\title{
Pressurized liquid extraction of active ingredients (ginsenosides) from medicinal plants using non-ionic surfactant solutions
}

\author{
Maggie P.K. Choi ${ }^{a}$, Kelvin K.C. Chan ${ }^{b}$, Hei Wun Leung ${ }^{b}$, Carmen W. Huie ${ }^{a, b, *}$ \\ ${ }^{a}$ Department of Chemistry, Hong Kong Baptist University, 224 Waterloo Road, Kowloon Tong, Hong Kong \\ ${ }^{\mathrm{b}}$ Quality Research Laboratory, School of Chinese Medicine, Hong Kong Baptist University, Kowloon Tong, Hong Kong
}

Received 17 June 2002; received in revised form 24 September 2002; accepted 1 October 2002

\begin{abstract}
The feasibility of employing aqueous non-ionic surfactant solutions as an alternative solvent system in pressurized liquid extraction (PLE) is demonstrated for the first time using the roots of American ginseng as model solid samples. When compared to the use of pure water or methanol, the presence of a common non-ionic surfactant (Triton X-100) in water at a concentration above its critical micelle concentration was shown to enhance the amount of pharmacologically active ingredients (ginsenosides) extracted from ginseng roots. The advantages of using aqueous non-surfactant solutions were also demonstrated by comparing extraction performances between ultrasonic-assisted extraction and PLE methods. Furthermore, the combination of PLE and cloud point extraction was shown to be a new and effective approach for the rapid sample preconcentration of herbal materials prior to analysis by high-performance liquid chromatography.
\end{abstract}

(C) 2002 Elsevier Science B.V. All rights reserved.

Keywords: Pressurized liquid extraction; Extraction methods; Cloud point extraction; Ginsenosides; Surfactants; Saponins

\section{Introduction}

The use of conventional sample preparation methods, such as Soxhlet extraction and sonication, for the extraction of analytes from solid materials suffers from a variety of disadvantages, including long extraction time, labor intensive procedures, large amount of organic solvents, unsatisfactory extraction efficiency and/or poor reproducibility [1,2]. During the past few years, the advent of a new extraction method known as pressurized liquid extraction (PLE; Dionex trade name ASE, for accelerated solvent

*Corresponding author. Fax: +852-3411-7348.

E-mail address: cwhuie@net1.hkbu.edu.hk (C.W. Huie). extraction), has shown good promise in overcoming some of the major drawbacks encountered in traditional extraction methods, mainly by taking advantage of the ability of liquid solvents at elevated temperatures and pressures in enhancing or accelerating extraction efficiency and/or kinetics [3].

Since the introduction of the first commercial PLE instrument a few years ago, the application of this technique has been focused on the extraction of environmental pollutants present in soil matrix, sewage sludge, sediments and fly ash [4,5]. However, more recently, the distinct advantages of PLE, such as significantly reduced extraction time and low solvent volume requirement, are being exploited in diverse areas, including biology, pharmaceuticals and foodstuffs [6]. An interesting and important new 
application area of PLE is in the extraction of chemical constituents from plants or herbal materials [6-14]. For instance, the use of water as solvent for the PLE of Taxol from the bark of yew tree has been shown to be more effective than other extraction methods [11]. Also, PLE has been recently applied for the extraction of various chemical ingredients, such as berberine [12], aristolochic acids [13] and ginsenosides [14], from medicinal plants.

In PLE the selection of suitable solvent systems is a key factor in the optimization of the extraction process [2,3]. Depending on the extraction conditions, such as the physiochemical properties of the analytes, chemical composition of the sample matrix, particle size of the samples, and extraction temperature/pressure, the extraction solvent can dramatically affect the extraction efficiency, kinetics as well as selectivity. In this paper, the feasibility and advantages of employing a new class of solvent system, i.e. aqueous non-ionic surfactant solutions, for the rapid and effective PLE of analytes from solid materials-active ingredients (ginsenosides) from the roots of American ginseng (Panax quinquefolium) is demonstrated. It should be noted that although the unique properties of surfactants have been exploited in various areas of analytical chemistry [15-18], the use of aqueous surfactant solutions as alternative solvent systems in PLE has not been reported in the literature.

In the present work, the effectiveness of employing an aqueous solution containing a common nonionic surfactant (Triton X-100) as the extracting medium in PLE was evaluated by comparing with conventional extraction solvents (water and methanol) as a function of various experimental parameters, such as temperature, pressure and concentration of the surfactant, for the extraction of ginsenosides. Also, extraction performances were compared between PLE and a conventional sample preparation method (ultrasonic-assisted extraction) for the extraction of ginsenosides using aqueous non-ionic surfactant solutions, water and methanol-water mixture as the extraction solvent. Additionally, the advantage of using the cloud-point property of Triton $\mathrm{X}-100$ for the preconcentration of the ginsenosides prior to high-performance liquid chromatography (HPLC) analysis was demonstrated.

\section{Experimental}

\subsection{Chemicals}

American ginseng samples were purchased from a local herbal shop in Hong Kong. The ginsenoside standards $\left(\mathrm{Rg}_{1}, \mathrm{Re}, \mathrm{Rb}_{1}, \mathrm{Rc}\right.$, and $\left.\mathrm{Rd}\right)$ were obtained from the National Institute for the Control of Pharmaceutical and Biological Products (Beijing, China). The non-ionic surfactant, Triton X-100, was obtained from Sigma (St. Louis, MO, USA) and used as received without further purification. Ammonium sulfate (reagent grade) used for salt-induced cloud point phase separation was obtained from Junsei (Tokyo, Japan). HPLC-grade acetonitrile and methanol were purchased from Acros (Gee, Belgium). All aqueous solutions were prepared with doubly deionized water (DDI) from a Milli-Q system (Millipore, Bedford, MA, USA).

\subsection{Preparation of ginseng samples}

The roots of American ginseng were dried in a vacuum oven (Model 1400E, VWR Scientific Products, West Chester, PA, USA) set at $50{ }^{\circ} \mathrm{C}$ for $6 \mathrm{~h}$, then ground and sieved to produce samples with particle sizes in the range between 40 and 120 mesh (sieve opening: 425 to $125 \mu \mathrm{m}$ ). The dried ginseng powder was stored in a moisture-controlled cabinet.

\subsection{Pressurized liquid extraction}

PLE extractions were carried out using a Dionex ASE 200 system (Sunnyvale, CA, USA). To begin extraction, a $0.1-\mathrm{g}$ ginseng sample was placed in a stainless steel cell (volume $11 \mathrm{ml}$ ) and extracted with the particular solvent system (volume $20 \mathrm{ml}$ ). Various concentrations (w/v) of aqueous non-ionic surfactant solutions were prepared by weighing out an appropriate amount of Triton X-100 and dissolving in DDI water. The automatic extraction sequence began with the loading of the cell into the oven. Subsequently, the cell was heated at the preset extraction temperature and solvent was continuously pumped through the sample. When the cell was full of the extraction solvent, the cell was heated and pressurized for a fixed time to ensure that the sample reached thermal 
equilibrium. Afterward, a static period occurred during which the sample was extracted for $5 \mathrm{~min}$ at the preset extraction temperature and pressure. The total extraction time was ca. 10 min (heating +one static period). After the static step, the extract was allowed to flow into the collection vial and the sample was rinsed with a portion of fresh solvent (30\% of the cell volume) under low pressure. Finally, the remaining solvent was displaced with a purge gas (nitrogen) for a period of $90 \mathrm{~s}$ and the collection vial now contained all of the solvent and analytes extracted from the sample. All extracts obtained were filtered through a $0.45 \mu \mathrm{m}$ nylon filter prior to injection into the HPLC system.

\subsection{Ultrasonic-assisted extraction}

Indirect sonication method was employed for extractions, i.e. the ultrasound energy was delivered to the extracting solvent via an ultrasonic cleaning bath (model 8891, Cole-Parmer, Vernon Hills, IL, USA). A 0.1-g ginseng sample was mixed with 20 $\mathrm{ml}$ of the extraction solvent in a 40-ml reagent bottle. The bottle was then closed and placed in the sonic bath with temperature maintained at $50{ }^{\circ} \mathrm{C}$ for all extractions. Afterward, the ginseng extract was filtered using Whatman filter papers $(110 \mathrm{~mm}$ diameter), followed by additional filtration through nylon filters $(0.45 \mu \mathrm{m})$ prior to injection into the HPLC system.

\subsection{Cloud point extraction/preconcentration}

To initiate salt-induced cloud point preconcentration, $\sim 4 \mathrm{~g}$ of ammonium sulfate were added to a $10-\mathrm{ml}$ ginseng extract (containing 1\% Triton X-100) within a centrifuge tube. The sample solution was shaken for 2 min using a vortex mixer and a cloudy sample solution was obtained. Phase separation was then achieved via centrifugation for $10 \mathrm{~min}$ at 4000 rev./min (centrifuge: model Z300, Hermle Labortechnik, Gosheimer, Germany). For temperature-induced cloud point preconcentration, the ginseng extract was placed into a water bath with the temperature kept at $78{ }^{\circ} \mathrm{C}$ for $1 \mathrm{~h}$. Afterward, the sample solution was cooled to room temperature and the upper aqueous phase was removed. For both salt- and temperature-induced phase separation, the surfactant-rich phase containing the preconcentrated ginsenosides was diluted slightly with a small amount of methanol-water (1:2) to reduce its viscosity prior to direct injection into the HPLC system.

\subsection{Analysis of the extracts by HPLC and $U V$ absorbance detection}

A HP1100 modular HPLC system consisting of a manual injector (with $20 \mu \mathrm{l}$ sample loop) and diodearray detector was employed. The separation column $(4.6 \mathrm{~mm} \times 25 \mathrm{~cm})$ was packed with $10 \mu \mathrm{m} \mathrm{C}_{8}$ material. The HPLC mobile phase consisted of DDI water and acetonitrile mixed according to a linear gradient program as shown in Table 1. The flow-rate and detection wavelength were set at $1.6 \mathrm{ml} / \mathrm{min}$ and $202 \mathrm{~nm}$, respectively.

\section{Results and discussion}

\subsection{Comparison of different solvent systems for the extraction of ginsenosides}

In Fig. 1, the total peak area of the five major ginsenosides (triterpene saponins: $\mathrm{Rg}_{1}, \mathrm{Re}, \mathrm{Rb}_{1}, \mathrm{Rc}$ and $\mathrm{Rd}$ ), which has been reported to account for more than $90 \%$ of the saponin content of the ginseng roots [19], is plotted as a function of different solvent systems as well as extraction temperatures. When comparing water and water containing various concentrations of the non-ionic surfactant (Triton $\mathrm{X}-100$ ), it can be seen that at each of the three extraction temperatures, the total amount of ginsenosides extracted (in terms of total peak area) was

Table 1

Mobile phase gradient program for ginsenosides determination

\begin{tabular}{lcl}
\hline Time (min) & Water & Acetonitrile \\
\hline 0 & 79 & 21 \\
14 & 79 & 21 \\
31 & 62 & 38 \\
31.1 & 5 & 95 \\
61 & 5 & 95 \\
61.1 & 79 & 21 \\
90 & 79 & 21 \\
\hline
\end{tabular}




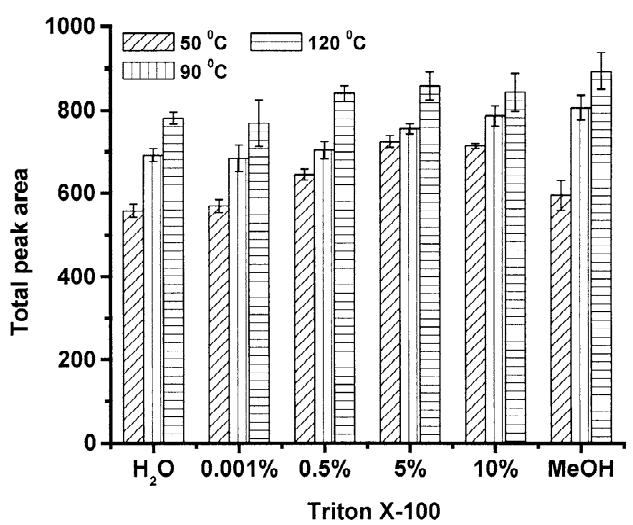

Fig. 1. Comparison of different solvent systems (water, aqueous Triton X-100 solutions and methanol) for the PLE of the five major ginsenosides at various extraction temperatures. The average value of each bar (total peak area of $\mathrm{Rg}_{1}, \mathrm{Re}, \mathrm{Rb}_{1}, \mathrm{Rc}$ and $\mathrm{Rd})$ was determined from the extraction of three samples. The extraction time and pressure for each solvent system were set at $10 \mathrm{~min}$ and 1500 p.s.i. $(1$ p.s.i. $=6.89476 \mathrm{kPa})$, respectively.

quite similar between these two solvent systems when the concentration of the surfactant was at $0.001 \%$, which is below the critical micelle concentration (CMC) of Triton X-100 (ca. 0.03\%) [16].

It can be seen from Fig. 1 that, however, when the surfactant concentrations exceeded the CMC, the total peak area of these major ginsenosides appeared to increase as a function of surfactant concentration. In particular, Fig. 1 shows that the total amount of saponins extracted at higher surfactant concentrations (5\% and $10 \%$ ) was larger than those obtained with water alone or Triton X-100 concentration at $0.001 \%$ (below the $\mathrm{CMC}$ ) for all three extraction temperatures. However, it is interesting to note that the increase in the total amount of ginsenosides extracted due to the addition of Triton X-100 in water at concentrations above the CMC (e.g. by comparing the total peak area between water and $5 \%$ or $10 \%$ Triton X-100) appeared to be much more pronounced at the lower extraction temperature (e.g. $50{ }^{\circ} \mathrm{C}$ compared to $120{ }^{\circ} \mathrm{C}$ ).

When compared to water, methanol is considered a better solvent for the extraction of ginsenosides from ginseng roots, largely due to the relatively high solubility of ginsenosides in methanol [20]. The data in Fig. 1 show that, at an extraction temperature of 90 and $120^{\circ} \mathrm{C}$, the use of methanol for the PLE of ginseng roots exhibited the largest total peak areas (e.g. slightly larger than those obtained with aqueous non-ionic surfactant solution at $5 \%$ or $10 \%$ Triton $\mathrm{X}-100)$. However, at a lower extraction temperature of $50{ }^{\circ} \mathrm{C}$, the total amount of saponins extracted with methanol was found to be significantly lower (e.g. clearly less than those obtained with $5 \%$ or $10 \%$ Triton X-100 but similar to those using water or water containing $0.001 \%$ Triton $\mathrm{X}-100$ at $50{ }^{\circ} \mathrm{C}$ ).

Importantly, Fig. 1 shows that at surfactant concentrations higher than the CMC $(0.5 \%$ to $10 \%$ Triton X-100), the total peak areas were larger than those using methanol at $50{ }^{\circ} \mathrm{C}$. When compared to water and methanol, the ability of aqueous non-ionic surfactant solutions in providing larger total peak areas at a lower extraction temperature may be related to the solubility-enhancement effect of the Triton X-100 micelles. For example, certain surfactants are known to increase the mass-transfer coefficient during the desorption of pollutants from soil to water, presumably due to better swelling of the soil organic matters and more complete diffusion of the solvent into the solid matrix [21].

The capability of the three different solvent systems in the PLE of two individual ginsenosides: Re and $R b_{1}$, which possess a relatively large difference in polarity, is illustrated in Fig. 2A and B. When compared to water and aqueous non-ionic surfactant solutions, Fig. 2A indicates that methanol was a poorer solvent for the PLE of the more hydrophilic ginsenoside ( $\mathrm{Re})$ at an extraction temperature of $50{ }^{\circ} \mathrm{C}$, but the peak area for Re increased dramatically when the extraction temperature was increased to $120{ }^{\circ} \mathrm{C}$. The reason that the use of water alone and water containing various concentrations of Triton $\mathrm{X}-100$ was more effective in the extraction of Re at the lower extraction temperature is likely in large part due to the higher solubility of Re in water and aqueous non-ionic surfactant solutions (compared to that of methanol at $50{ }^{\circ} \mathrm{C}$ ). It should be noted that, however, when the extraction temperature was increased from 50 to $120{ }^{\circ} \mathrm{C}$, the relative increase in peak area for Re obtained with water or aqueous Triton X-100 solutions as the solvent was considerably less than that with methanol (Fig. 2A).

In the case of the more hydrophobic ginsenoside $\left(\mathrm{Rb}_{1}\right)$, Fig. 2B shows that the effectiveness of extraction at 50 and $120^{\circ} \mathrm{C}$ was quite similar be- 

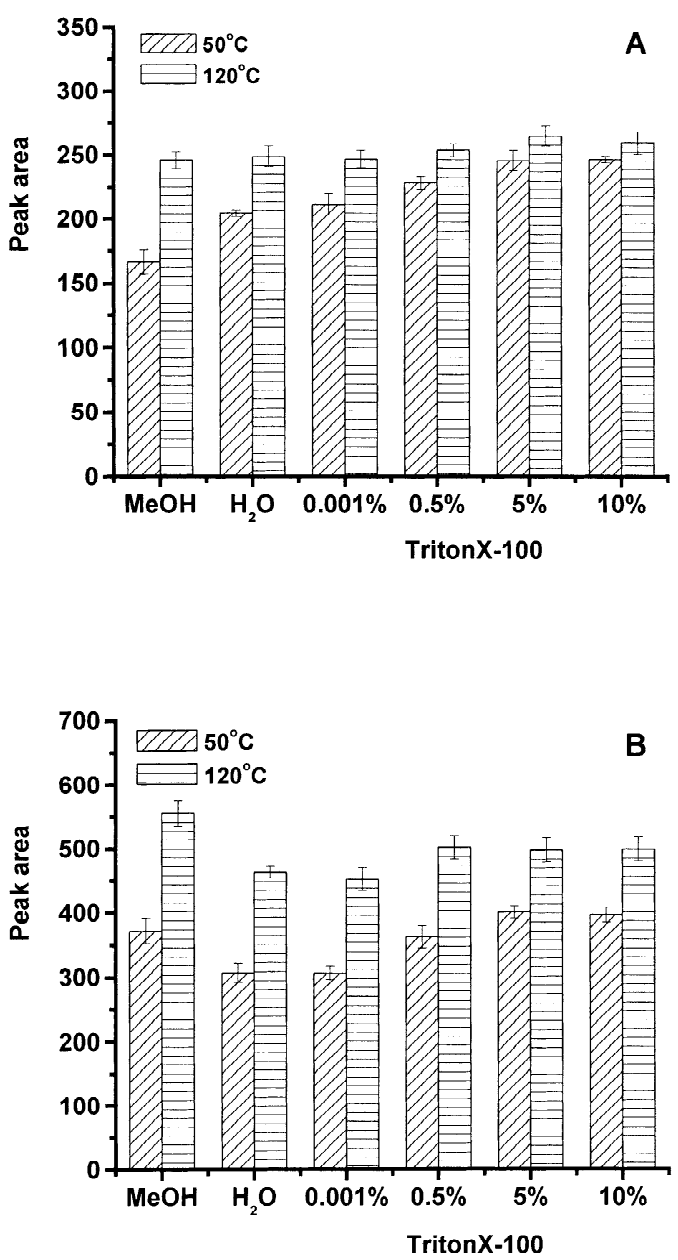

Fig. 2. Comparison of different solvent systems (water, aqueous Triton X-100 solutions and methanol) for the PLE of the ginsenoside (A) $\mathrm{Re}$ and (B) $\mathrm{Rb}_{1}$ at an extraction temperature of 50 and $120^{\circ} \mathrm{C}$, respectively. The extraction time and pressure for each solvent system were set at $10 \mathrm{~min}$ and 1500 p.s.i., respective1y. The average value of each bar was determined from the extraction of three samples.

tween water and water containing Triton X-100 at a concentration $(0.001 \%)$ below its CMC. At higher surfactant concentrations, however, it can be seen that $\mathrm{Rb}_{1}$ was extracted more effectively at an extraction temperature of 50 or $120^{\circ} \mathrm{C}$. When compared to methanol at the lower extraction temperature $\left(50{ }^{\circ} \mathrm{C}\right)$, Fig. $2 \mathrm{~B}$ shows that the peak area obtained with $5 \%$ or $10 \%$ Triton X-100 at the same extraction temperature was of similar magnitude. However, when the extraction temperature was in- creased to $120^{\circ} \mathrm{C}$, the use of methanol as the solvent provided the largest peak area for the extraction of $\mathrm{Rb}_{1}$.

\subsection{Effects of pressure and temperature using methanol and aqueous non-ionic surfactant solutions as the solvent system}

The ability of using high pressure and temperature to enhance or accelerate the extraction of ginsenosides using aqueous non-ionic surfactant solutions containing $1 \%$ Triton $\mathrm{X}-100$ as the solvent system was investigated. Fig. $3 \mathrm{~A}$ shows that at an extraction temperature of $50{ }^{\circ} \mathrm{C}$, the increase in pressure from 500 to 1500 p.s.i. led to an increase in the total peak area of all five major ginsenosides, but remained relatively constant from 1500 to 3000 p.s.i. On the other hand, when the extraction was performed at higher temperatures $\left(90\right.$ and $\left.120^{\circ} \mathrm{C}\right)$, the total peak area was increased as a function of temperature, but there appeared to be no significant changes throughout the entire pressure range of 500 to 3000 p.s.i.

In contrast, Fig. 3B shows that when using methanol as the extraction solvent, the extraction process appeared to be more sensitive to changes in pressure. For example, although the total peak area remained relatively constant from 500 to 3000 p.s.i. when the extraction temperature was set at $50{ }^{\circ} \mathrm{C}$, the total peak area appeared to exhibit a maximum at 1500 p.s.i. when extraction was performed at higher temperatures $\left(90\right.$ and $\left.120^{\circ} \mathrm{C}\right)$. At an extraction temperature of $200{ }^{\circ} \mathrm{C}$, however, the total peak area decreased significantly throughout the pressure range studied, especially at the lower pressure (500 p.s.i.), most likely in part due to thermal degradation of the various ginsenosides when the extraction temperature exceeded $120^{\circ} \mathrm{C}[20]$.

\subsection{Comparison of ultrasonic-assisted extraction and pressurized liquid extraction methods for the extraction of ginsenosides}

The use of ultrasound-assisted extraction (UAE) has been shown to be a simpler and more effective alternative to the classical extraction method of Soxhlet extraction (i.e. the use of refluxing boiling 

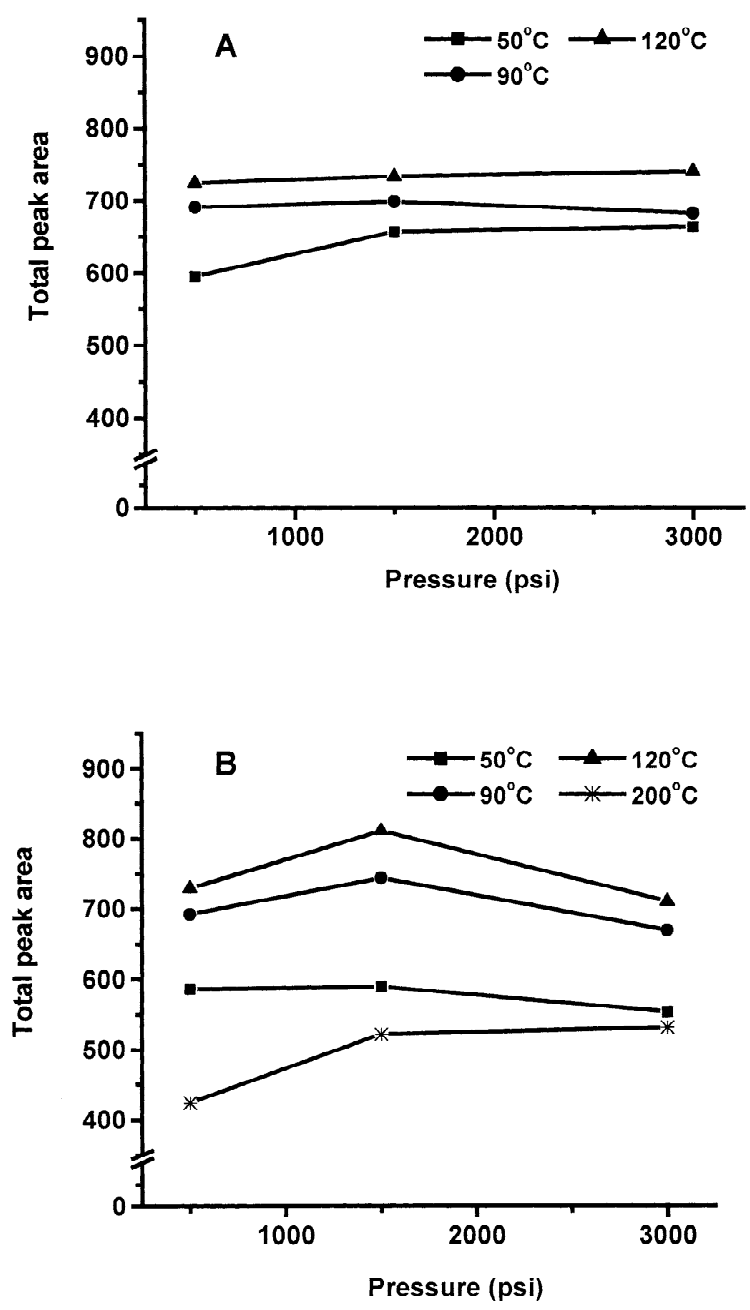

Fig. 3. Comparison of (A) $1 \%$ Triton X-100 and (B) methanol for the PLE of the five major ginsenosides (total peak area of $\mathrm{Rg}_{1}$, $\mathrm{Re}, \mathrm{Rb} \mathrm{b}_{1}, \mathrm{Rc}$ and $\mathrm{Rd}$ ) as a function of pressure and temperature. The extraction time for each of these solvents was set at $10 \mathrm{~min}$. solvent in a Soxhlet extractor) for the extraction of a variety of active ingredients from medicinal plants [22], including the extraction of saponins from ginseng roots [23]. Table 2 compares the effectiveness of using UAE and PLE for the extraction of the five major ginsenosides as a function of time, pressure and temperature using water as the extraction solvent. The results indicate that when the extraction time was reduced from $2 \mathrm{~h}$ to $10 \mathrm{~min}$, the individual as well as total peak area of the five ginseng saponins decreased markedly, especially for the more hydrophobic ginsenosides $\left(\mathrm{Rb}_{1}, \mathrm{Rc}\right.$ and $\mathrm{Rd})$. Thus, it is clear that longer extraction time is required for more complete UAE of the less watersoluble saponins when using water as the solvent.

On the other hand, the data in Table 2 indicate that when PLE was employed as the extraction method using the same extraction time and temperature (10 min and $50{ }^{\circ} \mathrm{C}$, respectively), there appeared to be an increase in the relative amounts of ginsenosides extracted, especially for the more non-polar saponins. The increase in the amounts of ginsenosides extracted is more obvious when the extraction temperatures were higher. At 90 and $120^{\circ} \mathrm{C}$, it is important to note that the peak areas for the various ginsenosides (using an extraction time of only $10 \mathrm{~min}$ ) were found to be near or slightly higher than those obtained using UAE at an extraction time of $2 \mathrm{~h}$.

Using $1 \%$ Triton $\mathrm{X}-100$ as the solvent in UAE, the data in Table 3 show that the total peak area of the saponins also decreased when the extraction time was reduced from $2 \mathrm{~h}$ to $10 \mathrm{~min}$; however, the individual peak area of the more hydrophobic ginsenosides $\left(\mathrm{Rb}_{1}, \mathrm{Rc}\right.$ and $\left.\mathrm{Rd}\right)$ remained relatively large

Table 2

Comparison of ultrasonic-assisted extraction (UAE) and pressurized liquid extraction (PLE) method for the extraction of five major ginsenosides using water as the solvent

\begin{tabular}{|c|c|c|c|c|c|c|c|c|c|}
\hline \multirow[t]{2}{*}{ Method } & \multirow[t]{2}{*}{ Time } & \multirow{2}{*}{$\begin{array}{l}\text { Temperature } \\
\left({ }^{\circ} \mathrm{C}\right)\end{array}$} & \multirow[t]{2}{*}{ Pressure $^{\mathrm{a}}$} & \multicolumn{6}{|c|}{ Normalized peak area } \\
\hline & & & & $\operatorname{Rg}_{1}$ & $\operatorname{Re}$ & $\mathrm{Rb}_{1}$ & $\mathrm{Rc}$ & $\mathrm{Rd}$ & Total $^{\mathrm{b}}$ \\
\hline UAE & $2 \mathrm{~h}$ & 50 & $1 \mathrm{~atm}$ & $1 \pm 0.05$ & $1 \pm 0.01$ & $1 \pm 0.04$ & $1 \pm 0.08$ & $1 \pm 0.05$ & $1 \pm 0.02$ \\
\hline UAE & $10 \mathrm{~min}$ & 50 & $1 \mathrm{~atm}$ & $0.80 \pm 0.03$ & $0.90 \pm 0.04$ & $0.68 \pm 0.04$ & $0.50 \pm 0.03$ & $0.30 \pm 0.01$ & $0.75 \pm 0.03$ \\
\hline PLE & $10 \mathrm{~min}$ & 50 & 1500 p.s.i. & $0.80 \pm 0.04$ & $0.95 \pm 0.02$ & $0.84 \pm 0.02$ & $0.51 \pm 0.04$ & $0.56 \pm 0.02$ & $0.85 \pm 0.02$ \\
\hline PLE & $10 \mathrm{~min}$ & 90 & 1500 p.s.i. & $0.85 \pm 0.03$ & $0.95 \pm 0.05$ & $1.07 \pm 0.03$ & $0.82 \pm 0.05$ & $1.15 \pm 0.05$ & $1.00 \pm 0.03$ \\
\hline PLE & $10 \mathrm{~min}$ & 120 & 1500 p.s.i. & $0.99 \pm 0.05$ & $1.01 \pm 0.04$ & $1.20 \pm 0.03$ & $1.13 \pm 0.08$ & $1.39 \pm 0.07$ & $1.12 \pm 0.04$ \\
\hline
\end{tabular}

\footnotetext{
${ }^{\mathrm{a}}$ Conversion factors for pressure: $1 \mathrm{~atm}=101.325 \mathrm{kPa}$; 1 p.s.i. $=6.89476 \mathrm{kPa}$.

${ }^{\mathrm{b}}$ The total peak area was calculated from the addition of the individual peak areas from the five major ginsenosides and was an average value of the extraction of three samples.
} 
Table 3

Comparison of ultrasonic-assisted extraction (UAE) and pressurized liquid extraction (PLE) method for the extraction of five major ginsenosides using $1 \%$ Triton $\mathrm{X}-100$ as the solvent

\begin{tabular}{|c|c|c|c|c|c|c|c|c|c|}
\hline \multirow[t]{2}{*}{ Method } & \multirow[t]{2}{*}{ Time } & \multirow{2}{*}{$\begin{array}{l}\text { Temperature } \\
\left({ }^{\circ} \mathrm{C}\right)\end{array}$} & \multirow[t]{2}{*}{ Pressure } & \multicolumn{6}{|c|}{ Normalized peak area } \\
\hline & & & & $\overline{\mathrm{Rg}_{1}}$ & $\mathrm{Re}$ & $\mathrm{Rb}_{1}$ & $\mathrm{Rc}$ & $\mathrm{Rd}$ & Total $^{\mathrm{a}}$ \\
\hline UAE & $2 \mathrm{~h}$ & 50 & $1 \mathrm{~atm}$ & $1 \pm 0.05$ & $1 \pm 0.07$ & $1 \pm 0.02$ & $1 \pm 0.03$ & $1 \pm 0.04$ & $1 \pm 0.03$ \\
\hline UAE & $10 \mathrm{~min}$ & 50 & $1 \mathrm{~atm}$ & $0.72 \pm 0.05$ & $0.95 \pm 0.03$ & $0.89 \pm 0.03$ & $0.84 \pm 0.04$ & $0.70 \pm 0.03$ & $0.90 \pm 0.02$ \\
\hline PLE & $10 \mathrm{~min}$ & 50 & 1500 p.s.i. & $0.87 \pm 0.04$ & $0.99 \pm 0.02$ & $0.90 \pm 0.01$ & $0.90 \pm 0.05$ & $0.70 \pm 0.001$ & $0.92 \pm 0.003$ \\
\hline PLE & $10 \min$ & 90 & 1500 p.s.i. & $0.89 \pm 0.04$ & $1.03 \pm 0.02$ & $0.95 \pm 0.02$ & $0.91 \pm 0.05$ & $0.91 \pm 0.02$ & $0.98 \pm 0.01$ \\
\hline PLE & $10 \mathrm{~min}$ & 120 & 1500 p.s.i. & $0.90 \pm 0.05$ & $1.04 \pm 0.02$ & $1.00 \pm 0.06$ & $1.05 \pm 0.06$ & $0.92 \pm 0.05$ & $1.01 \pm 0.04$ \\
\hline
\end{tabular}

${ }^{\mathrm{a}}$ The total peak area was calculated from the addition of the individual peak areas from the five major ginsenosides and was an average value of the extraction of three samples.

when compared to those of water alone as the extraction solvent (Table 2). When using PLE as the extraction method, the increase in pressure (from $1 \mathrm{~atm}$ to 1500 p.s.i.) appeared to provide a slight increase in the individual as well as total peak area when compared to those of UAE (10 min extraction), and this increase was more pronounced at a higher extraction temperature of 90 and $120^{\circ} \mathrm{C}$.

The above data indicate that the addition of Triton $\mathrm{X}-100$ in water significantly improved the extraction of the more non-polar ginsenosides $\left(\mathrm{Rb}_{1}, \mathrm{Rc}\right.$ and $\mathrm{Rd})$ at the lower extraction temperature $\left(50^{\circ} \mathrm{C}\right)$, using either UAE or PLE as the extraction method. Table 4 shows that similar results were obtained when using a binary mixture of methanol and water (7:3) as the solvent instead of $1 \%$ Triton X-100. It should be noted that the use of methanol-water mixtures, instead of pure methanol, has been shown to provide enhanced extraction performances for the isolation and purification of various ginseng saponins from ginseng roots [20].

\subsection{Cloud-point preconcentration prior to HPLC analysis of ginsenosides}

A relatively large number of publications can be found in the literature concerning the determination of ginsenosides using various HPLC methods $[14,24-27]$. In recent years, the unique cloud-point behavior of non-ionic surfactants (i.e. the ability of aqueous non-surfactant solutions in separating into two distinct phases, a surfactant-rich phase and a bulk aqueous phase, when the critical temperature is exceeded) has attracted much interest for its capability in preconcentrating a variety of analytes prior to HPLC analysis without the need of using relatively toxic organic solvents such as methanol [2831]. For example, Fang et al. recently reported the use of ultrasonic-assisted extraction, followed by cloud point extraction for the HPLC determination of ginsenosides and American ginseng [31]. To the best of our knowledge, however, the combined use of PLE and cloud point methodology for the extraction

Table 4

Comparison of ultrasonic-assisted extraction (UAE) and pressurized liquid extraction (PLE) method for the extraction of five major ginsenosides using a mixture of methanol and water (7:3) as the solvent

\begin{tabular}{|c|c|c|c|c|c|c|c|c|c|}
\hline \multirow[t]{2}{*}{ Method } & \multirow[t]{2}{*}{ Time } & \multirow{2}{*}{$\begin{array}{l}\text { Temperature } \\
\left({ }^{\circ} \mathrm{C}\right)\end{array}$} & \multirow[t]{2}{*}{ Pressure } & \multicolumn{6}{|c|}{ Normalized peak area } \\
\hline & & & & $\mathrm{Rg}_{1}$ & $\mathrm{Re}$ & $\mathrm{Rb}_{1}$ & $\mathrm{Rc}$ & $\mathrm{Rd}$ & Total $^{\mathrm{a}}$ \\
\hline $\mathrm{UAE}$ & $2 \mathrm{~h}$ & 50 & $1 \mathrm{~atm}$ & $1 \pm 0.01$ & $1 \pm 0.04$ & $1 \pm 0.02$ & $1 \pm 0.05$ & $1 \pm 0.04$ & $1 \pm 0.01$ \\
\hline UAE & $10 \mathrm{~min}$ & 50 & $1 \mathrm{~atm}$ & $0.79 \pm 0.03$ & $0.93 \pm 0.01$ & $0.85 \pm 0.02$ & $0.86 \pm 0.03$ & $0.79 \pm 0.03$ & $0.89 \pm 0.01$ \\
\hline PLE & $10 \mathrm{~min}$ & 50 & 1500 p.s.i. & $0.80 \pm 0.05$ & $0.93 \pm 0.03$ & $0.94 \pm 0.02$ & $0.81 \pm 0.02$ & $0.89 \pm 0.05$ & $0.93 \pm 0.01$ \\
\hline PLE & $10 \mathrm{~min}$ & 90 & 1500 p.s.i. & $1.04 \pm 0.07$ & $0.97 \pm 0.01$ & $1.00 \pm 0.01$ & $0.98 \pm 0.04$ & $0.97 \pm 0.05$ & $0.99 \pm 0.01$ \\
\hline PLE & $10 \mathrm{~min}$ & 120 & 1500 p.s.i. & $1.10 \pm 0.04$ & $0.98 \pm 0.01$ & $1.04 \pm 0.04$ & $1.13 \pm 0.09$ & $1.11 \pm 0.05$ & $1.02 \pm 0.03$ \\
\hline
\end{tabular}

\footnotetext{
${ }^{\mathrm{a}}$ The total peak area was calculated from the addition of the individual peak areas from the five major ginsenosides and was an average
} value of the extraction of three samples. 
and preconcentration of analytes has not been reported in the literature.

Fig. 4A shows the HPLC chromatogram of a sample obtained from the PLE of ginseng roots using
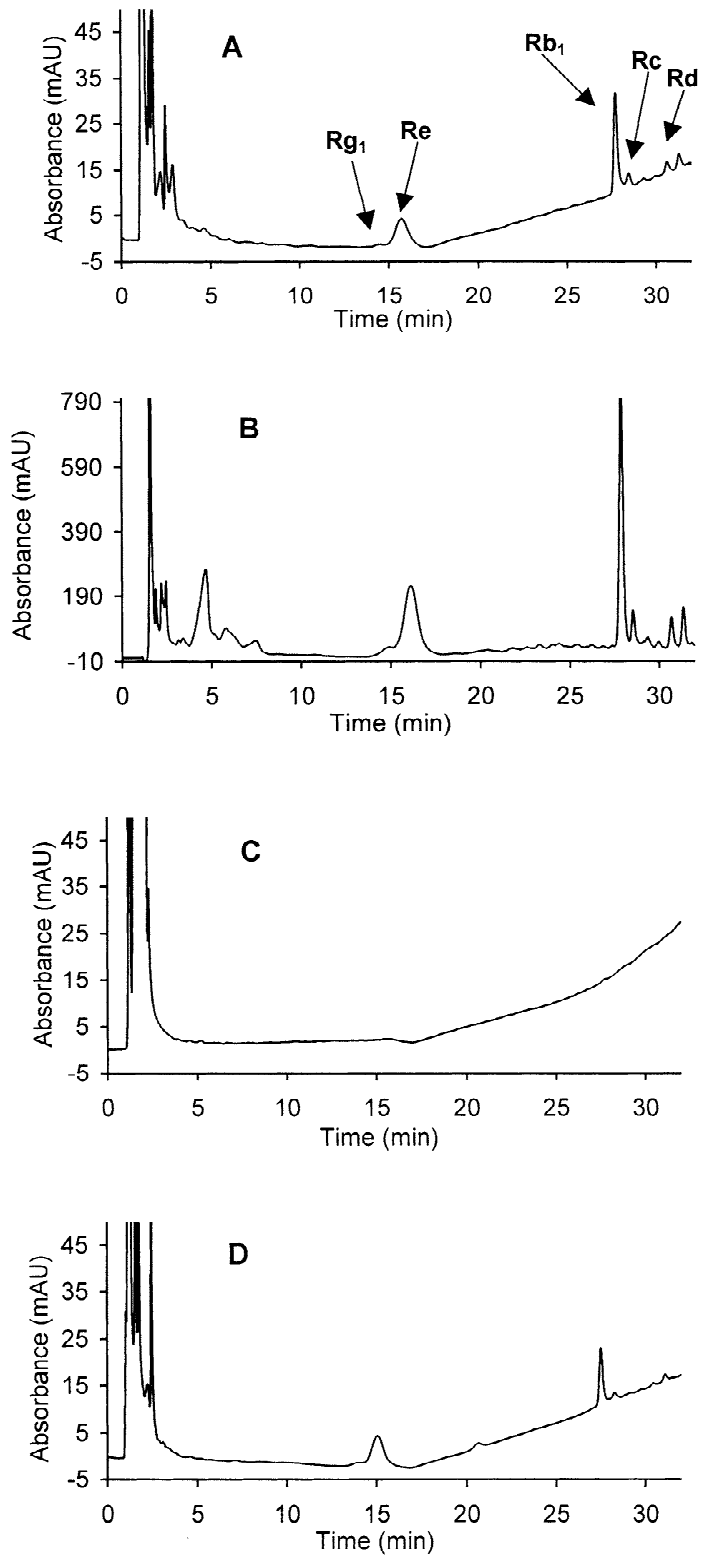

Fig. 4. HPLC chromatograms of (A) $1 \%$ Triton X-100 solution containing the ginsenosides prior to cloud point phase separation (non-preconcentrated sample), (B) the surfactant-rich phase containing ginsenosides (preconcentrated sample due to salt-induced phase separation of $1 \%$ Triton X-100), (C) the bulk aqueous phase after salt-induced phase separation and (D) the bulk aqueous phase after temperature-induced phase separation.
$1 \%$ Triton X-100 (without inducing the cloud-point phenomenon). Similar chromatograms were also obtained when pure water or a binary mixture of methanol-water (7:3) was used as the solvent instead. To increase detection sensitivity for the various ginsenosides, preconcentration via cloud point phase separation can be achieved by heating the sample solution above the critical temperature, but this approach is mostly limited to the extraction and preconcentration of hydrophobic substances. On the other hand, it has been demonstrated in recent studies [30,31] that the addition of appropriate salting-out agents into the sample solutions to induce the cloud point phenomenon allowed for the efficient extraction of both hydrophobic and hydrophilic compounds into the surfactant-rich phase.

When compared to Fig. 4A, Fig. 4B (larger absorbance scale) shows that a marked increase in detection sensitivity was obtained for all five major ginsenosides (an average enhancement factor of ca. 35 in terms of peak area) by injecting an aliquot of the preconcentrated sample (upper surfactant-rich phase obtained via salt-induced cloud point extraction of the $1 \%$ Triton X-100 sample solution). The effectiveness of using salting-out and temperatureinduced method was evaluated by examining the amount of ginsenosides remaining in the bulk aqueous phase as shown in Fig. 4C and D, respectively. When compared to Fig. 4A and C, Fig. 4D clearly shows that a relatively large amount of ginseng saponins, especially the more hydrophilic ginsenosides, still remained in the aqueous phase when temperature was used as a means to induce cloud point phase separation.

It should be noted that, as shown in Table 1, a solvent gradient with a time period of ca. 30 min was used to separate the various major ginsenosides; another 30 min was used to wash the surfactant and perhaps other UV-absorbing substances from the column, followed by another $30 \mathrm{~min}$ for re-equilibration. It was found that at least 20 to $30 \mathrm{~min}$ of washing with a solvent consisting of acetonitrilewater (95:5) was required to obtain a relatively stable baseline. On the other hand, due to the fact that a rather aged column was used in the present HPLC study, a relatively lengthy re-equilibration time was employed in trying to obtain the best precision possible for the extraction data shown in Tables 2-4. As such, it is conceivable that both the 
washing and re-equilibration time could be reduced, especially if a newer column, a non-UV absorbing non-ionic surfactant as the extractant, and/or a more selective detector is available for usage [28]. In fact, the coupling of cloud-point extraction with HPLC is a relatively well-established method and a "lengthy" elution time for washing/re-conditioning the column has not been cited in the literature as a requirement or necessity [28-30].

\section{Conclusions}

When compared to the use of water alone or methanol as the solvent, the addition of a common non-ionic surfactant (Triton X-100) in water at concentrations above the CMC was found to enhance the PLE of ginsenosides from the roots of American ginseng, albeit the extent of this increase appeared to be relatively small at higher extraction temperatures. At lower extraction temperatures (e.g. $50{ }^{\circ} \mathrm{C}$ as shown in Figs. 1 and 2A), however, the enhancement was found to be considerably higher. Such an enhancement is likely related to the ability of the surfactant micelles (compared to water alone or methanol) in increasing the solubility of the ginsenosides and the rate of mass transfer at lower extraction temperatures. Therefore, an important advantage of using aqueous non-ionic surfactant solutions as a solvent system in PLE, as opposed to the use of plain aqueous or organic solvents as reported for PLE methods reported in the literature for the extraction and analysis of chemical substances in medicinal plants [6-14], is that extraction could be carried out at lower temperatures without a significant compromise in extraction efficiency, and thus avoiding the degradation of thermally unstable ingredients in plant materials.

When using aqueous solutions as extraction solvents in UAE or PLE, the addition of Triton X-100 (above CMC) in water should allow for a considerable saving in extraction time while maintaining relatively high extraction efficiency for the extraction of both hydrophobic and hydrophilic ginsenosides. For example, UAE was recently evaluated as a simpler and more effective alternative to conventional extraction methods for the isolation of ginsenosides from various types of ginseng, including American ginseng [23]. The results indicated that when using UAE and different aqueous-based extraction solvents (e.g. water with $10 \%$ methanol) for the extraction of the various major ginsenosides, extraction efficiencies obtained with $2 \mathrm{~h}$ of UAE was comparable to those achieved by $8 \mathrm{~h}$ of Soxhlet extraction. However, the extraction yields were found to decrease dramatically (e.g. reduced by 20 to $40 \%$ ) when the sonication period was set between 15 and $45 \mathrm{~min}$. In the present study, the data shown in Tables 2 and 3 indicate that the presence of Triton X-100 in water did enhance the UAE or PLE of various ginsenosides, especially for the more hydrophobic species, and the effectiveness of extraction obtained with $2 \mathrm{~h}$ of UAE was comparable to those achieved by only $10 \mathrm{~min}$ of PLE at higher extraction temperatures.

Although PLE has been recently developed for the extraction of ginsenosides in medicinal plants and health supplements prior to HPLC analysis, the extraction solvent employed was methanol [14]. The use of aqueous surfactant solutions instead of organic solvents such as methanol for sample preparation should be safer, less expensive and more environmentally friendly [15-18]. Perhaps more importantly, from an analytical standpoint, the use of the cloud-point property of non-ionic surfactants is capable of effecting rapid preconcentration of various ginsenosides into a relatively small volume surfactant-rich phase, which is one of the major advantages of coupling cloud-point extraction to various chromatographic techniques when compared to other sample preconcentration methods [28-31]. Since the use of traditional medicines/herbal products is becoming more popular worldwide [6-14] and the lack of simple, rapid and reliable analytical methods for the analysis of herbal products appears to be a major cause of quality-related problems [32], the coupling of PLE and cloud point extraction could be employed as a new and effective approach for the extraction and preconcentration of various pharmacologically active compounds from medicinal plants prior to chromatographic analysis.

\section{Acknowledgements}

Financial support from the Research Grant Council of the UGC (HKBU 2056/98P) is gratefully acknowledged. 


\section{References}

[1] R.E. Majors, LC-GC 14 (1996) 88.

[2] V. Camel, Analyst 126 (2001) 1182.

[3] B.E. Richter, B.A. Jones, J.L. Ezzel, N.L. Porter, N. Avdalovic, C. Pohl, Anal. Chem. 68 (1996) 1033.

[4] S. Lundstedt, B. van Bavel, P. Haglund, M. Tysklind, L. Öberg, J. Chromatogr. A 883 (2000) 151.

[5] I. Windal, D.J. Miller, E. De Pauw, S.B. Hawthorne, Anal. Chem. 72 (2000) 3916.

[6] B. Benthin, H. Danz, M. Hamburger, J. Chromatogr. A 837 (1999) 211.

[7] J. Suomi, H. Sirén, K. Hartonen, M.-L. Riekkola, J. Chromatogr. A 868 (2000) 73.

[8] E.-S. Ong, S.-O. Woo, Y.-L. Yong, J. Chromatogr. A 313 (2000) 57.

[9] C.W. Huie, Anal. Biol. Chem. 373 (2002) 23.

[10] B. Kaufmann, P. Christen, Phytochem. Anal. 13 (2002) 105.

[11] F. Kawamura, Y. Kikuchi, T. Ohira, M. Yatagai, J. Nat. Prod. 62 (1999) 244.

[12] G.W. Schieffer, K. Pfeiffer, J. Liq. Chromatogr. Relat. Technol. 24 (2001) 2415.

[13] E.S. Ong, S.O. Woo, Electrophoresis 22 (2001) 2236.

[14] H.K. Lee, H.L. Koh, E.S. Ong, S.O. Woo, J. Sep. Sci. 25 (2002) 160.

[15] G.L. McIntire, Crit. Rev. Anal. Chem. 21 (1990) 257.

[16] W.L. Hinze, E. Pramauro, Crit. Rev. Anal. Chem. 24 (1993) 133.
[17] A. Bianco Prevot, M. Gulmini, V. Zelano, E. Pramauro, Anal. Chem. 73 (2001) 3790.

[18] Q. Fang, M. Du, C.W. Huie, Anal. Chem. 73 (2001) 3502.

[19] W.A. Court, J.G. Hendel, J. Elmi, J. Chromatogr. A 755 (1996) 11.

[20] M.L. Anderson, D.P. Burney, J. AOAC Int. 81 (1998) 1005.

[21] D. Sahoo, J.A. Smith, Environ. Sci. Technol. 31 (1997) 1910.

[22] M. Vinatoru, Ultrason. Sonochem. 8 (2001) 303.

[23] J. Wu, L. Lin, F.-T. Chau, Ultrason. Sonochem. 8 (2001) 347.

[24] X.M. Wang, T. Sakuma, E. Asafu-Adjaye, G.K. Shiu, Anal. Chem. 71 (1999) 1579.

[25] N. Fuzzati, B. Gabetta, K. Jayakar, R. Pace, G. Ramaschi, F. Villa, J. AOAC Int. 83 (2000) 820.

[26] Q.C. Ji, M.R. Harkey, G.L. Henderson, M.E. Gershwin, J.S. Stern, R.M. Hackman, Phytochem. Anal. 12 (2001) 320.

[27] W.K. Li, J.F. Fitzloff, J. Pharm. Pharmacol. 53 (2001) 1637.

[28] R. Carabias-Martínez, E. Rodríguez-Gonzalo, B. Moreno Cordero, J.L. Pérez-Pavón, C. García-Pinto, E. Fernández Laespada, J. Chromatogr. A 902 (2000) 251.

[29] D.S. Bai, J.L. Li, S.B. Chen, B.H. Chen, Environ. Sci. Technol. 35 (2001) 3936.

[30] S.R. Sirimanne, J.R. Barr, D.G. Patterson Jr., Anal. Chem. 68 (1996) 1556.

[31] Q. Fang, H.W. Yeung, H.W. Leung, C.W. Huie, J. Chromatogr. A 904 (2000) 47.

[32] L. Picker, Am. Health 15 (1996) 70. 